\title{
Vibration Energy Harvesting Dampers for Massive Large-Scale Objects
}

\author{
George Nerubenko $^{1}$, David Flowers ${ }^{2}$,Ivan Biliuk $^{3}$ \\ ${ }^{1}$ NER*MAR Limited \\ 270 Palmdale Drive, Toronto, ON, M1T 3N8 Canada \\ info@mega-automation.ca \\ ${ }^{2}$ Flowers Engineering \\ 1791 Highway, Indian Harbour Beach, FL 32937 USA \\ d.c.flowers@ieee.org \\ ${ }^{3}$ National University of Shipbuilding \\ 9 Ave. Geroev,Nikolaev,54025, Ukraine \\ ivan.bilyuk@nuos.edu.ua
}

\begin{abstract}
In this paper, the new way of a protection from the intensive vibrations of massive large-scale huge objects like masts, towers, bridges, ships and others are described. The innovative idea is to combine in one vibration suppressing apparatus two functions: the ability of sufficientvibration damping combined to energy harvesting in broad range of actual frequencies. The invented by authors Vibration Energy Harvesting Damper is used for mitigation of undesired vibrations and generating of the additional electrical power. The perspective feature of proposed device is the effective vibration damping in broad range of frequencies due to implementation of a control device, while that device is keeping automatically the changing natural frequency of a device equals to current frequency of object's oscillations. The mathematical simulation analysis based on parameters of the real objects is developed. The experiments arranged on test platform illustrate the compliance with theoretical predictions, and efficiency of proposed tool operating mainly in low frequency interval.
\end{abstract}

Keywords: oscillation / vibration damping, energy harvesting, towers, bridges, ships.

\section{Introduction}

The massive huge heavy large-sized structures and objects (like masts, towers, ships, bridges, skyscrapers, etc.) are subjected to the different types of dynamical loads (see for instance [1]-[4] and others). One of the results of dynamic loads is the various kinds of object's oscillations. The practice demonstrates that the frequencies of above mentioned disturbances are sitting mainly in range $0.0 \mathrm{~Hz}-20.0 \mathrm{~Hz}$. One of the most effective instruments for suppressing such oscillations is Tuned Mass Damper (TMD) - the device consisting of mass reacting on oscillating object movements through specifically designed springing element [5]. The idea of combining the damping properties of TMD with energy harvesting was developed by authors in $[6,7]$ and that device was called the Vibration Energy Harvesting Damper (VEHD). The energy harvesting is the technology allowing capture, convert and transmit the certain amount of wasted power $[8,9]$. The results of VEHD development for different industrial applications were discussed in [10] - [13]. The installation of VEHD into massive large-sized structures is dictated by two interconnected reasons: required reduction of intensive oscillations and proper usage of damped vibration energy. The current study is the attempt to introduce the VEHD technology in these objects.

\section{Common Properties of the Massive Large-Scale Objects}

A general evaluation of maximal power Po lost by a certain oscillating object and possibly extracted from it could be done approximately based on a formula from the theory of vibrations (see for instance [10] $-[12]$ ): Po = $0.5 \beta \mathrm{A}_{\mathrm{m}}{ }^{2} \boldsymbol{\omega}^{2}$, where $\mathbf{A m}$ is a resonance amplitude of an object, $\boldsymbol{\omega}$ is a resonance frequency, $\boldsymbol{\beta}$ is a viscous damping coefficient. For example, for a mast, taking the conservative numbers for amplitude as $0.1 \mathrm{~m}$, and resonance frequency as $3 \mathrm{~Hz}$, and average number for viscous damping coefficient like $14100 \mathrm{~kg} / \mathrm{s}$, the value of the possible maximal extracted power would be $25 \mathrm{~kW}$. That example demonstrates the tremendous opportunities not discovered and not implemented in the current engineering solutions. 
The discussed objects in this study have some common properties. Let's explain it using the real examples.

The authors were involved in one of the CN Tower (Toronto, Canada) project in 1998.CN Tower is the 553 meters tower. On the top there are two premises (1850 ft above sea level elevation and $1900 \mathrm{ft}$ above sea level elevation). These premises contain two TMD with total weight of 18 ton. The TMD rings are elevated at 15 meters above each other. It is needed to mention that the top antenna could sway up to $1.07 \mathrm{~m}$ from centre, and the authors register a frequency of top oscillation as approximately $1 \mathrm{~Hz}$.

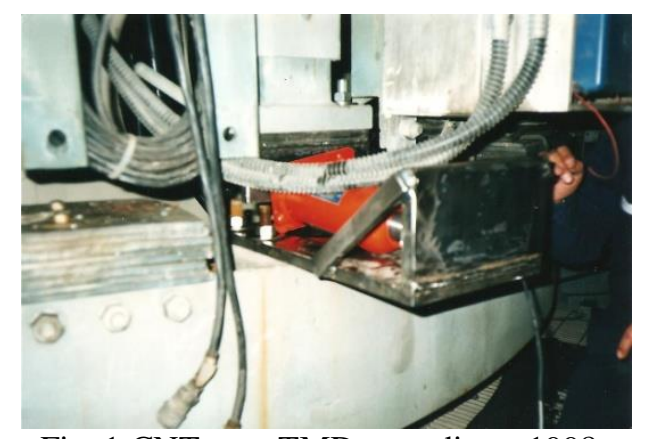

Fig. 1:CNTower TMD recordings. 1998.

It was established during 1998 research work that the maximum amplitude of " $1850 \mathrm{ft}$ " room oscillations was $0.8 \mathrm{~m}$. The photo of lower TMD ring fragment (sitting in " $1850 \mathrm{ft}$ " room) is in Fig.1. Trying to calculate the possible maximal extracted power using the published numbers, one would get 6,000 megawatt.

One of the authors took part in tests of the 50,000-tonore bulk carrier Zoya Kosmodemyanskaya (see Fig. 2). She is $215.40 \mathrm{~m}$ long, main diesel engine power is $8830 \mathrm{~kW}$ and travels at a speed of 15 knots. The energy estimates shows that she could generate potentially up to $5000 \mathrm{~kW}$ on 2 meter wave during pitching.

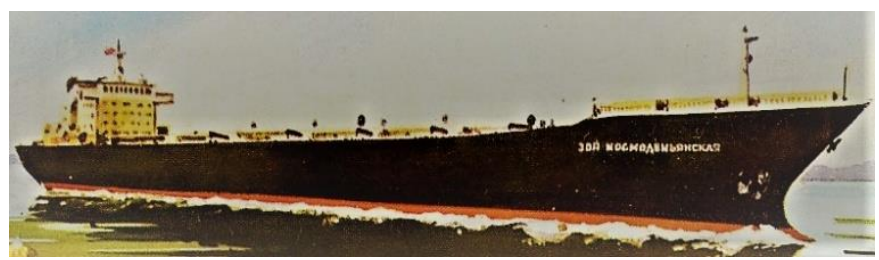

Fig. 2:Bulkcarrier ZoyaKosmodemyanskaya.

The authors were invited to make selection and design of TMD system for Australian pedestrian footbridge in 2006. The footbridge total length was $178 \mathrm{~m}$, and weight was $505432 \mathrm{~kg}$. The predicted calculated natural frequency of vertical vibrations was $2.7 \mathrm{~Hz}$, and an acceleration of $3 \mathrm{~m} / \mathrm{s}^{2}$. The estimates show that the possible maximal extracted power is about $80 \mathrm{~kW}$.

The presented examples illustrate the key common figures of considered huge heavy large-sized objects: they have a tremendous weight, sizes over 100 meters. The natural frequencies of their oscillations are usually in range $0.0-20.0 \mathrm{~Hz}$, while amplitude of vibrations could be up to 1 meter and more. These objects have a magnificent potential for the electricity generation (the energy harvesting).

\section{VEHD Modelling}

The analysis of design and structural features of the considered object shows that the rectilinear operating VEHD should be used successfully. The basic graphical model of rectilinear operating VEHD ([7], [10] - [12]) is shown in Fig. 3.

The object $M$ is vibrating along coordinate axis $X$ as shown in Figure 3. The mass of vibrating object is M. VEHD consists of two major parts: TMD and Alternator. TMD having mass $\mathrm{m}$ is attached to VEHD's body by means of a spring 2 
with some dissipative damping properties simulated by element 3. The alternator portion consists of pick-up coil 4 and a magnet 5. A magnet 5 is attached firmly to the mass $m$ of TMD. TMD together with a magnet have to have own resonant frequency $\omega$ o equal to object operational frequency $\omega$. The amplitude of mass $\mathbf{m}$ vibrations would be maximal in this case, and "productivity" of Alternator would be the maximal as well. The traditional rectilinear VEHD could be adjusted for single natural frequency $\omega_{\mathrm{o}}$, for example $\omega^{2}=\mathrm{k} 1 / \mathrm{m}=\omega_{\mathrm{o}}^{2}$, where $\mathrm{k} 1$ is the constant stiffness coefficient of springing element 2 in Figure 3. Now, if there is need to adjust $\mathrm{k} 1 / \mathrm{m}=\omega_{0}^{2}$ to variable $\omega$, it must be used the special device providing metering of the changing values $\omega$, and adjusting stiffness coefficient of spring k1. In that case the magnitudes of k1 would be variable.

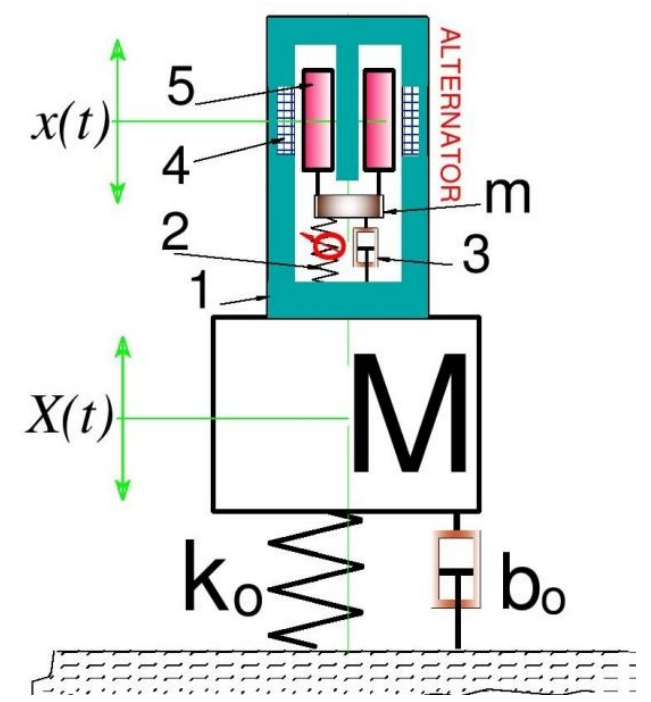

Fig. 3: The graphical model of VEHD installed on the object.

Using the modelling it would be supposed that the simplified object has a mass $M$ supporting by a spring having a stiffness ko and a viscosity factor bo. It is assumed that the object is the oscillating system with one degree of freedom. The body 1 of VEHD is attached firmly to an object. It is assumed (for simplicity) that the object could vibrate in one direction $\mathrm{X}(\mathrm{t})$ only, where $\mathrm{t}$ is a time. The outer casing of VEHD is firmly attached to an object, and the mass of a casing is included in M. TMD together with a magnet $\mathrm{m}$ are connected to a casing by means of springs having the stiffness k1 and viscosity factor $b 1$. A mass $m$ is vibrating in the same direction as a mass $M$. The coordinate of vibrations of $m$ is $x(t)$. So the system "an object equipped by TMD" is the oscillating system with two degrees of freedom. The electromagnetic subsystem is inducing a current $\mathrm{i}$, and $\mathrm{L}$ is an inductance, $\mathrm{R}$ is the electrical resistance, $\mathrm{R}=\mathrm{Rc}+\mathrm{Rd}$, where $\mathrm{Rc}$ is coil resistance and $\mathrm{Rd}$ is load resistance. The VEHD dynamics is described by a system of ordinary differential equations [8] - [12]:

$$
\begin{gathered}
\mathrm{MX}+\mathrm{boX}+\mathrm{koX}+\mathrm{m} \omega^{2}(\mathrm{X}-\mathrm{x})+\mathrm{b} 1\left(\mathrm{X}^{\prime}-\mathrm{x}^{\prime}\right)=\mathrm{H} \sin (\omega \mathrm{t}) \\
\mathrm{m \textrm {X } ^ { \prime }}-\mathrm{m} \omega^{2}\left(\mathrm{X}^{\prime} \mathrm{x}\right)-\mathrm{b} 1\left(\mathrm{X}^{\prime}-\mathrm{x}^{\prime}\right)+\mathrm{F} \mathrm{i}=\mathbf{0} \\
\mathrm{Li}+\mathrm{Ri}-\mathrm{F}\left(\mathrm{x}^{\prime}-\mathrm{X}^{\prime}\right)=0
\end{gathered}
$$

where $\mathbf{H}$ is the magnitude of external force, $\boldsymbol{\omega}$ is the frequency of external force, ${ }^{`}=\mathrm{d} / \mathrm{dt}$, and $\mathbf{F}$ is the linkage factor. The linkage factor could be determined in most cases as $\mathbf{F}=\mathbf{B L s}$, where $\mathbf{B}$ is field flux and $\mathbf{L s}$ is characteristic size. One can see that the system of equations (1) - (3) includes the specific features - the automatic frequency tuning $\omega_{0}=\omega_{\text {. }}$.

The stability analysis of a system (1) - (3) demonstrates that there are some restrictions for selections of the stiffness ko and k1. 
Introducing $\mathrm{C}=\mathrm{k} 1 / \mathrm{ko}$, and $\mathrm{a}=\mathrm{m} / \mathrm{M}$ it is possible to build the graph showing the stable zone for these parameters selection (see Fig.4).

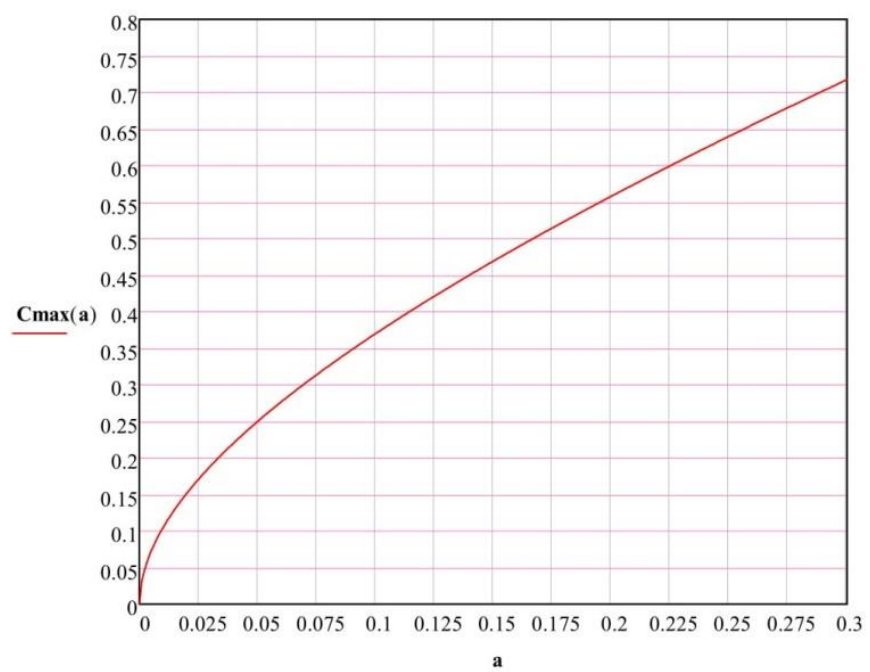

Fig. 4: Permitted zone for selecting $\mathrm{C}=\mathrm{k} 1 / \mathrm{ko}$ vs. $\mathrm{a}=\mathrm{m} / \mathrm{M}$.

It is needed to note that the interval of ratio a is within $0-0.3$ (accordingly to real values) in Fig.4, and the stable zone is restricted by a horizontal line $\mathrm{C}=0$, up to the red solid curve $\mathrm{Cmax}$.

\section{Dynamical and Power Analysis of a System Equipped by VEHD}

The dynamical analysis of a considered object equipped by VEHD should be demonstrated numerically and graphically using the system of the ordinary differential equations (1) - (3). Assume that the object has the following characteristics: $M=1 \mathrm{~kg}, \mathrm{bo}=25 \mathrm{~kg} / \mathrm{s}, \mathrm{ko}=4000 \mathrm{~kg} / \mathrm{s} 2, \mathrm{H}=3 \mathrm{~kg} \mathrm{~m} / \mathrm{s}^{2}$. The natural frequency will be $10.07 \mathrm{~Hz}$. The reason of selecting object's mass equals to $1 \mathrm{~kg}$ will be discussed later.

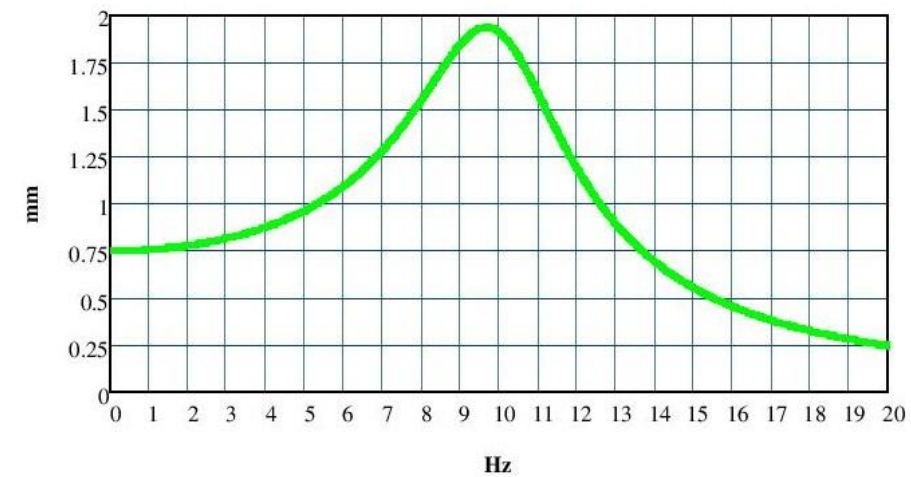

A

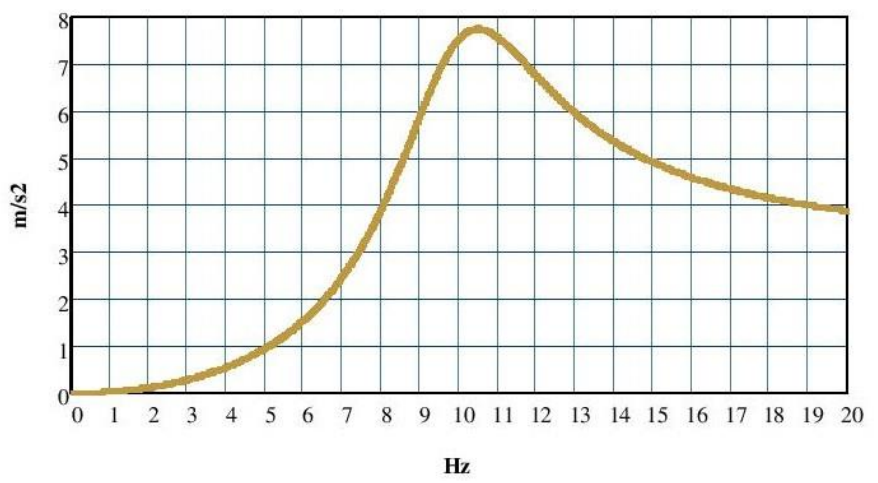

B

Fig. 5: $\mathrm{M}=1 \mathrm{~kg}, \mathrm{H}=3 \mathrm{~kg} \mathrm{~m} / \mathrm{s}^{2}$. A. Amplitude-Frequency Characteristics of the oscillating object. No VEHD; B. Acceleration-Frequency Characteristics of the oscillating object. No VEHD.

The amplitude - frequency characteristic for object's vertical vibration is shown in a Figure 5A by the green solid curve. The abscissa horizontal axis is for excitation frequency in $\mathrm{Hz}$ and an ordinate vertical axis is for amplitude in mm. There is the acceleration - frequency characteristic shown in a Figure 5B by the light brown solid curve; here the ordinate 
vertical axis is for acceleration in $\mathrm{m} / \mathrm{s} 2$.The amplitude of the vertical vibration resonance is $1.936 \mathrm{~mm}$, and the peak acceleration is $7.742 \mathrm{~m} / \mathrm{s}^{2}$. The estimates show that the possible maximal generated power is 0.18 Watt.

Let's select the VEHD with TMD - Alternator having mass as $6 \%$ of object's mass, stiffness $\mathrm{k} 1=\mathrm{m} \omega^{2}$ and all other parameters are subjected to values recommended in [10] - [12]. The amplitude -frequency characteristics of object having such VEHD are shown in a Figure 6. The amplitude - frequency characteristic of an object without installed VEHD is reflected still by the green solid curve in a Figure 6A (similar to plot in a Figure 5A). The blue dotted curve in a Figure 6A stands for damped vibrations of an object when VEHD installed. The amplitude of system with VEHD in resonance is equal to $0.995 \mathrm{~mm}$. The rate of amplitude reduction at initial system resonances is 1.95 times. The peak of amplitude of system equipped by selected VEHD is $1.199 \mathrm{~mm}$ at $8 \mathrm{~Hz}$.

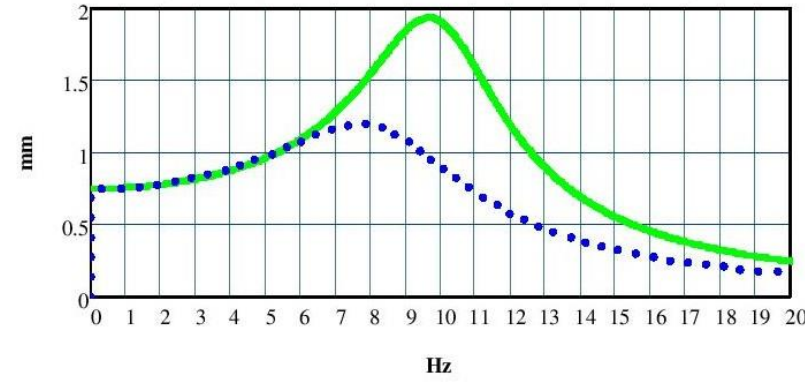

A

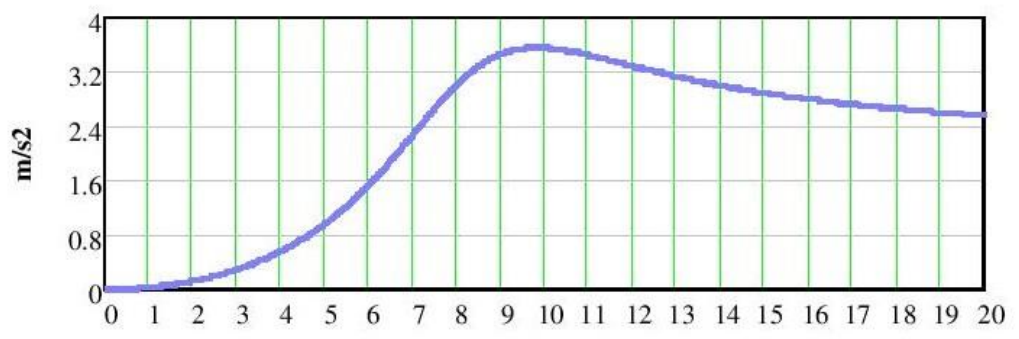

$\mathrm{Hz}$

Fig. 6:M=1 kg, H=3 kg m/s $\mathrm{s}^{2}$. A. Amplitude-Frequency Characteristics of the oscillating object. VEHD installed; B. AccelerationFrequency Characteristics of the oscillating object. VEHD installed.

The acceleration - frequency characteristic shown in Figure 6B by the blue solid curve has a peak of $3.56 \mathrm{~m} / \mathrm{s}^{2}$, and the rate of acceleration reduction is 2.17 times.

Let's build the TMD amplitude - frequency characteristic and VEHD induced power. These graphs are plotted in a Figure 7. TMD amplitude - frequency characteristic is shown by the dark brown dashed curve in a Figure 7A. The green curve in a Figure 7A is the equivalent of green curve in a Figure 6A characterizing the dynamical behaviour of an object without installed VEHD studied above, and blue dotted curve is the same as shown in a Figure 6A for damped vibrations of an object when VEHD installed. The purpose of presentation of these two curves on graph of a Figure 7A is to demonstrate the significant difference between amplitudes of object and TMD. The value of maximal TMD amplitude is $20.6 \mathrm{~mm}$ at $7.3 \mathrm{~Hz}$.

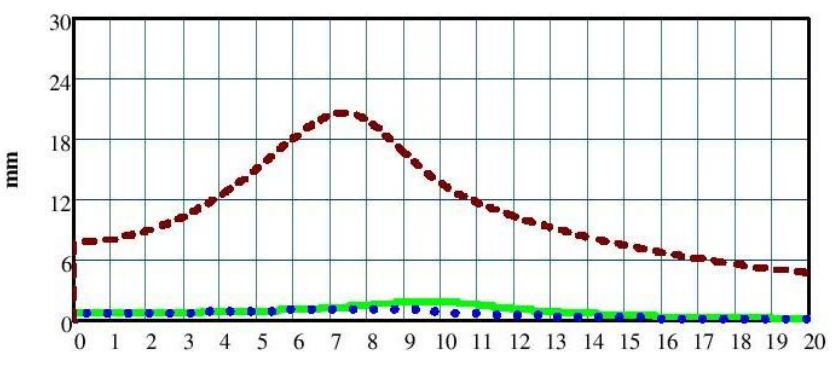

$\mathrm{Hz}$

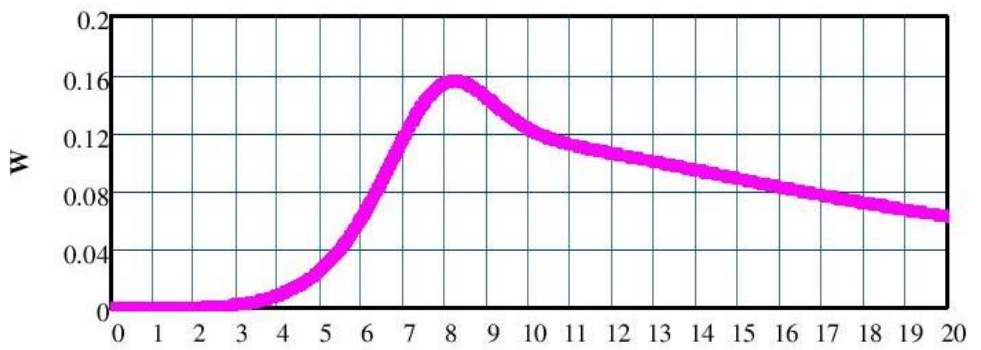

$\mathrm{Hz}$

A

Fig. 7:M=1 kg, H=3 kg m/s². A. Amplitude-Frequency Characteristics of the oscillating object. VEHD installed; B. Generated power by installed VEHD. 
The power behaviour would be presented by magenta solid curve as shown in Figure 7B. The abscissa horizontal axis stands for excitation frequency in $\mathrm{Hz}$ and ordinate vertical axis is for useful generated power in Watt. The shape and location of magenta solid curve (peak of useful power) in Figure 7B is repeating the shape and location of brown dashed curve in Figure 7A. It is the confirmation that Alternator's parameters are dictated at some extent by dynamics of TMD. The maximal value of generated power is 0.155 Watt at $8.3 \mathrm{~Hz}$. Analysis of obtained results demonstrates that the selected object having $1 \mathrm{~kg}$ mass and experiencing the resonance vibration at $10 \mathrm{~Hz}$ with the peak acceleration of $7.742 \mathrm{~m} / \mathrm{s}^{2} \mathrm{should}$ get reduced vibration and acceleration if to mount the proposed VEHD with TMD - Alternator mass equals to $6 \%$ of object's mass. The reduction of resonance acceleration is in 2.17 times, and the maximum magnitude of generated power is 0.155 Watt.

It is interesting to analyze the behaviour of considered system when the amplitude of external force would raised in 10 times, so $\mathrm{H}=30 \mathrm{~kg} \mathrm{~m} / \mathrm{s} 2$. The amplitude - frequency characteristic for that case is shown in a Figure $8 \mathrm{~A}$ by the green solid curve. The observer can see that the amplitude of the vertical vibration resonances $19.355 \mathrm{~mm}$ now, or in 10 times more than in previous case when $\mathrm{H}=3 \mathrm{~kg} \mathrm{~m} / \mathrm{s} 2$. Suppose that all other initial parameters equal to earlier selected (including TMD - Alternator mass equals to $6 \%$ of object's mass), and examine the useful generated power behaviour. The useful generated power is presented in Figure $8 \mathrm{~B}$, and one can see that the maximum number of generated power is 15.5 Watt now, increasing is in 100 times in comparison to case when $\mathrm{H}=3 \mathrm{~kg} \mathrm{~m} / \mathrm{s} 2$.

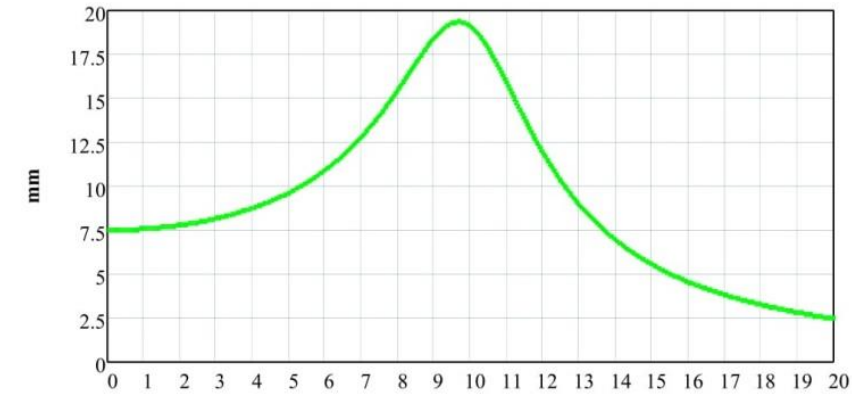

$\mathbf{H}$

A

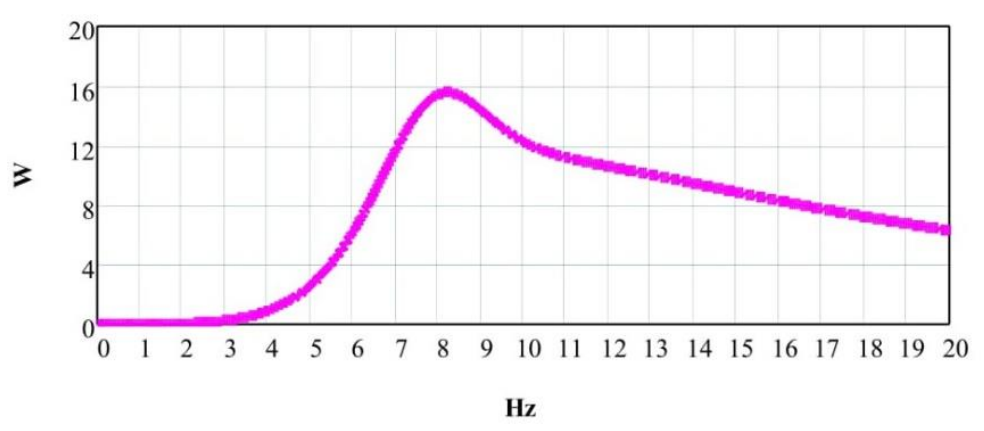

B

Fig. 8: $\mathrm{M}=1 \mathrm{~kg}, \mathrm{H}=30 \mathrm{~kg} \mathrm{~m} / \mathrm{s}^{2}$. A. Amplitude-Frequency Characteristics of the oscillating object. No VEHD; B. Generated power by installed VEHD.

The engineer could state at this point that the increase of external force magnitude leads to proportional raise of amplitudes of an object, and the useful generated power is raised quadratically.

Now let's do the similar modelling, simulation and estimates for the case when a mass of object is equal to $10 \mathrm{~kg}$, and all other initial parameters would be selected in such way that received new amplitude - frequency characteristics should be similar to previously obtained for the case when object's mass $1 \mathrm{~kg}$ and $\mathrm{H}=3 \mathrm{~kg} \mathrm{~m} / \mathrm{s}^{2}$. Furthermore it is possible obtain the same amplitude - frequency characteristics as for $1 \mathrm{~kg}$ object if to nominate TMD - Alternator mass equals to $6 \%$ of 10 $\mathrm{kg}$ object's mass with correspondingly selected parameters of an alternator. The only difference is that the power characteristics are changed. The new power curve would be presented in Figure 9. 


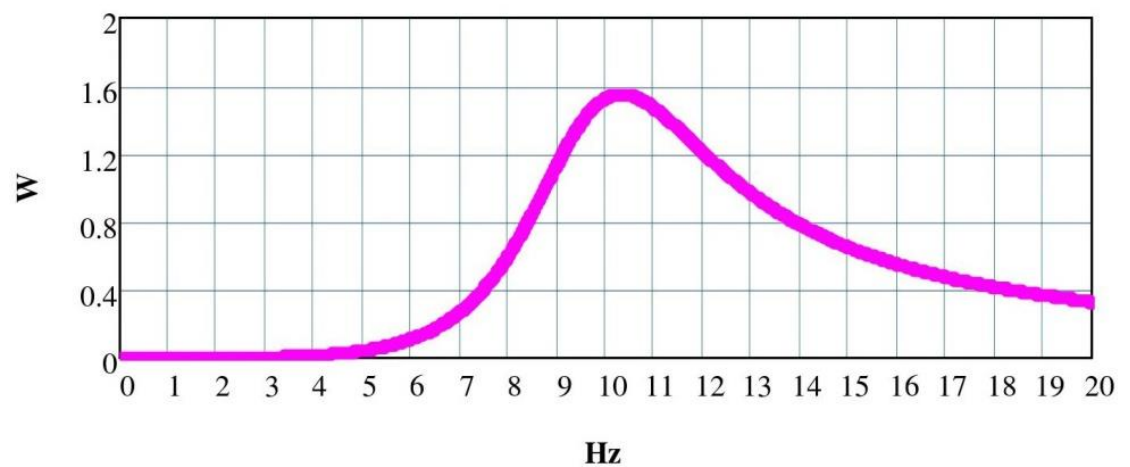

Fig. 9: $\mathrm{M}=10 \mathrm{~kg}, \mathrm{H}=3 \mathrm{~kg} \mathrm{~m} / \mathrm{s}^{2}$. Generated power by installed VEHD.

The maximum value of generated power is $1.55 \mathrm{~W}$ att at $10.4 \mathrm{~Hz}$. In other words, the generated power increased in 10 times in the discussed case in comparison to $1 \mathrm{~kg}$ and $\mathrm{H}=3 \mathrm{~kg} \mathrm{~m} / \mathrm{s} 2$ case.

The selection of a object's mass equal to $100 \mathrm{~kg}$, and keeping the above mentioned procedures and rules for all other parameters choice, one can get the following results: all dynamical characteristics would be the same as for case $1 \mathrm{~kg}$ object and $10 \mathrm{~kg}$ object, but the maximum value of generated power be15.5 Watt (in 10 times more than for case with 10 $\mathrm{kg}$ object). Hence, the heavier object could generate for free more electrical power.

\section{Tests of a System Equipped by VEHD}

The dynamical tests were organized using the test platform with a shaker [11] (see Figure 10).

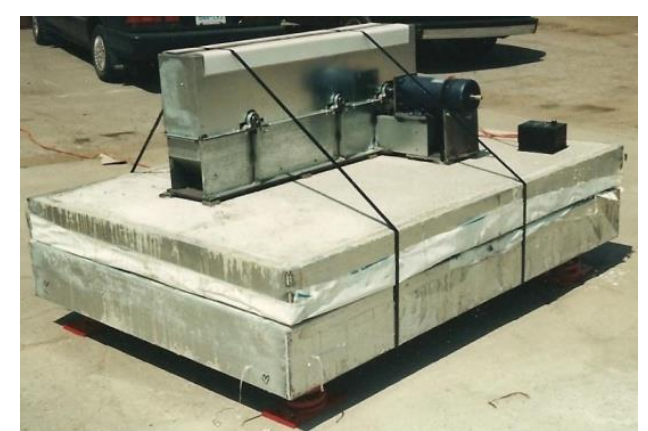

Fig. 10:The equipped test platform.

The rectangular flat platform has a re-adjustable mass. Each of four corners of a platform is sitting of springs. These springs are equipped by low pressure hydraulic cylindrical dampers. There is a shaker on the upper face of a platform. The shaker has the driving device - DC motor equipped by the speed controller. The shaker produces the stable state sinusoidal force acting in the vertical direction with a range of frequencies $1.5 \mathrm{~Hz}-20 \mathrm{~Hz}$.

The designed and manufactured platform for tests has total mass (platform and shaker) $300 \mathrm{~kg}$. The design of a platform was made in line with previously selected and recommended numbers. The test results were recorded and analyzed. The tests of such fabricated system show the following values: the amplitude of the platform's vertical resonance vibration is $2 \mathrm{~mm}$ (see records in a Figure 11 A and compare to a Figure 5A), and the peak acceleration is $7.7 \mathrm{~m} / \mathrm{s}^{2}$ at $10 \mathrm{~Hz}$. These numbers are very close to the predicted ones. 

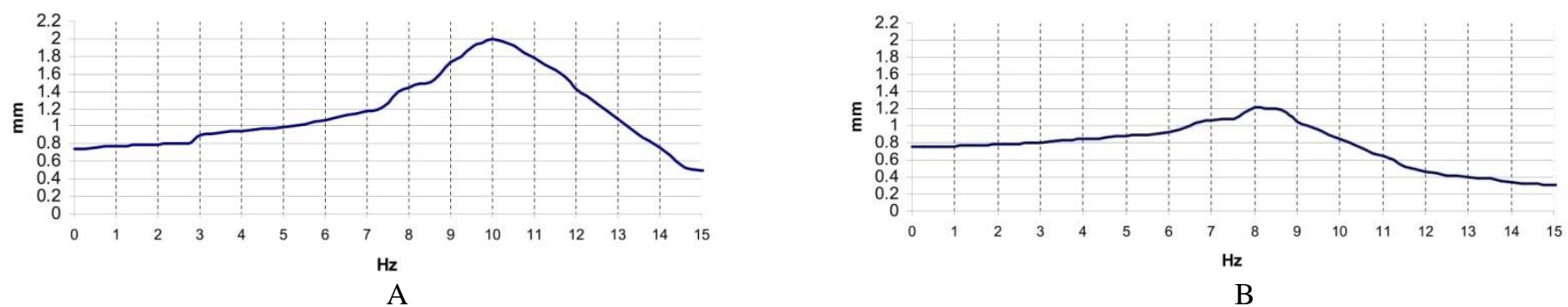

Fig. 11: The recorded displacements of tested platform. A. Platform only, no VEHD. B. Platform with VEHD.

The prototyping of VEHD was done in a following way. In accordance to a theory it must be one VEHD with TMD Alternator mass equals to $18 \mathrm{~kg}$ ( $6 \%$ of object's mass). It was decided to make six VEHD with TMD - Alternator mass equals to $3 \mathrm{~kg}$ and locate them underneath of a platform perimeter. The main characteristics of prototypes were based on values presented in modelling cases. VEHD was vertically installed. The amplitude of a system with VEHD in a resonance is equal to $0.82 \mathrm{~mm}$. The peak of amplitude of a system equipped by selected VEHD is $1.21 \mathrm{~mm}$ at $8.1 \mathrm{~Hz}$ (see records in Figure 11B, and compare to blue dotted curve in Figure 6A).The acceleration peak was of $3.45 \mathrm{~m} / \mathrm{s}^{2}$.The maximum value of generated power was recorded at all six prototypes and summarized. The total maximum value of generated power was 46.9 Watt. Let's compare that result to theoretical prediction. Theoretically it was studied the object having $100 \mathrm{~kg}$ mass, movable VEHD mass was $6 \mathrm{~kg}$, and produced maximum power of 15.5 Watt. Now suppose that an object has the increased mass in 3 times (300 kg like for tested platform). Hence installing VEHD with movable part of $18 \mathrm{~kg}$, the expected maximum power would be 46.5 Watt, so an accuracy of the theoretical prediction (comparing to obtained 46.9 Watt in tests) is $0.9 \%$.

\section{Conclusion}

Taking into consideration the natural properties of massive large-scale objects, namely the low frequency (usually not more than $20 \mathrm{~Hz}$ ) oscillations with significant displacements, it is understandable to equip them by Vibration Energy Harvesting Dampers achieving two goals: reduction of the vibration levels of an object by controlled tuned mass dampers, coupled with generation an additional portion of power which could be used for free.

The implementation of invented electromagnetic automatically controlled Vibration Energy Harvesting Dampers gives flexibility in applications: it provides a possibility to use them in such formats as focused on tuning damping, or focused on reasonable balance between tuning damping of an object oscillations and effectiveness of the wasted power usage, or focused on the effectiveness of using wasted power only.

The implementation of Vibration Energy Harvesting Dampers into structure of massive large-scale objects gives the opportunity generates significant amounts of additional green power (up to megawatts) for free.

There are a lot of positive side effects of introducing invented Vibration Energy Harvesting Dampers into massive large-scale objects like a reduction of fatigue in structures, arranging independent electricity generation station, feeding wireless autonomous electronic units, comfort level improvements and much more.

\section{References}

[1] T. Knudsen, T. Bak, S. Tabatabaeipour, "Detection of excessive wind turbine tower oscillations fore-aft and sideways," American Control Conference (ACC), pp. 5795-5800,2012.

[2] E.Kreuzer, M. Pick. "Dynamics of Ship-Motion,"PAMM Proc. App. Math. Mech., vol. 3, pp. 84-87, 2003.

[3] P. Persson, P. Austrell, P. Kirkegaard, "Analysis of wind-induced vibrations in high-rise buildings," Inter-Noise 2016, Hamburg, pp. 7650-7661.

[4] P. J. McKenna, C. O'Tuama, "Large torsional oscillations in suspension bridges visited again: Vertical forcing creates torsional response," Am. Math. Monthly, vol. 108, pp. 738-745, 2001. 
[5] S. Eliseev, G. Nerubenko,"Tuned Mass Dampers,"NAUKA, Novosibirsk, 1982.

[6] G. Nerubenko, "Torsional Vibration Damper of a Rotating Shaft," US Patent 7464 800,2008.

[7] G. Nerubenko, D. Flowers et al, "Vibration Energy Harvesting Damper," U.S. Patent 10644579, May 5, 2020.

[8] S. Priya, D. L. Inman, "Energy Harvesting Technologies", Sprinder, Verlag, 2009.

[9] F. Cottone, "Energy Harvesting: introduction,"NiPS Summer School,Fiuggi, Italy,pp. 50, 2015.

[10] A. Bograd, G. Nerubenko, C. Nerubenko, "Energy Harvesting in Vehicle's Drive," Proceedings of the 2nd International Conference of Energy Harvesting, Storage, and Transfer (EHST'18), Niagara Falls, Canada,2018.

[11] G. Nerubenko et al, "Tuned mass dampers as energy harvesters for railways," Proceedings of ISMA2018 and USD2018. Leuven, Belgium, pp. 1689-1702.

[12] G. Nerubenko, "Vibration Energy Harvesting Damper in Vehicle Driveline," SAE Technical Paper, 2019.

[13] L. Marian and A. Giaralis, "The tuned mass-damper-inerter for harmonic vibrations suppression, attached mass reduction, and energy harvesting, "Smart structures and systems, vol. 19, no. 6, pp. 665-678,2017. 